\title{
Pin 1 contributes to cervical tumorigenesis by regulating cyclin D1 expression
}

\author{
HONGYU LI ${ }^{1,2}$, SHIXUAN WANG ${ }^{*}$, TAO ZHU ${ }^{1}$, JINHUA ZHOU ${ }^{1}$, QIAN XU ${ }^{1}$, YUNPING LU ${ }^{1}$ and DING MA ${ }^{*}$ \\ ${ }^{1}$ Cancer Biology Research Center, Tongji Hospital, Tongji Medical College, Huazhong University of \\ Science and Technology, Wuhan, Hubei 430030; ${ }^{2}$ Department of Obstetrics and Gynecology, \\ the Third Affiliated Hospital, Zhengzhou University, Zhengzhou, Henan 450052, P.R. China
}

Received March 10, 2006; Accepted May 30, 2006

\begin{abstract}
The prolyl isomerase Pin1, which specifically catalyzes conformational changes in certain proline-directed phosphorylation sites, is thought to be a critical catalyst for multiple oncogenic pathways. However, little is known about the role of Pin 1 in human cervical cancer. Our previous study showed that Pin 1 was overexpressed in cervical cancer tissues as well as cell lines. In this study, whether Pin 1 is involved in cervical oncogenesis by regulating cyclin D1 was explored and the potential of Pin1-targeted gene silencing in inhibiting cellular growth and tumorigenicity in cervical cancer was investigated. A Pin1-directed shRNA and a sense Pin1 plasmid were constructed, and then the effects of the shRNA and the sense plasmid on HeLa cells were evaluated. The results showed that Pin 1 directly regulated cyclin D1 levels. In addition, silencing Pin1 with RNAi significantly reduced cancer cell proliferation, colony formation, and strongly enhanced the apoptosis of HeLa cells. It is suggested that Pin 1 may contribute to cervical tumorigenesis by regulating cyclin D1 expression and Pin1 may serve as a promising molecular target for diagnostics and therapeutics in cervical cancer.
\end{abstract}

\section{Introduction}

Phosphorylation of proteins on serine or threonine residues preceding proline (pSer/Thr-Pro) is a major regulatory mechanism in cell proliferation and transformation. Interestingly, the pSer/Thr-Pro motifs in proteins exist in two distinct cis and trans conformations, whose conversion rate is normally reduced on phosphorylation, but is catalyzed

Correspondence to: Dr Ding Ma, Department of Cancer Biology Research Center, Tongji Hospital, Tongji Medical College, Huazhong University of Science and Technology, 1095 Jiefang Ave., Wuhan, Hubei 430030, P.R. China

E-mail:dma@tjh.tjmu.edu.cn; dingma424@yahoo.com

${ }^{*}$ Contributed equally

Key words: Pin1, overexpression, cyclin D1, RNAi, cervical cancer specifically by the prolyl isomerase Pin 1 . Pin 1 catalyzes conformational changes in certain key proline-directed phosphorylation sites and functions as a pivotal catalyst for multiple oncogenic pathways (1-8). Recently, it has been shown that Pin 1 is strikingly overexpressed in many different human cancers (9). Pin1 plays a critical role in the transformation of mammary epithelial cells and closely associates with breast cancer grades $(8,10)$. Furthermore, Pin1 expression is an excellent independent prognostic marker in prostate cancer (11). These results suggest that the level and function of Pin1 are pivotal for cell proliferation and oncogenic signaling $(12,13)$.

Cyclin D1 is a proto-oncogenic cell cycle regulator of the G1/S check-point in the cell cycle and plays an important role in the development of many cancers $(14,15)$. A series of in vitro and in vivo studies demonstrate a close correlation between Pin1 and cyclin D1 (10,16-18). Pin1 regulates cyclin D1 gene expression by at least three different mechanisms $(10,19-24)$, and directly binds to and presumably isomerize the pThr 286-Pro motif of cyclin D1 thereby preventing its nuclear export and ubiquitin-mediated degradation, resulting in cyclin D1 stabilization (17).

Cervical cancer is closely associated with human papillomavirus (HPV) infection. But viral infection alone is not sufficient to initiate malignant transformation, additional genetic changes must occur in order to elicit malignant transformation (25). Although Pin1 plays an important role in the transformation of mammary epithelial cells (26), it is not known whether Pin1 expression is involved in cervical oncogenesis. In our previous study, Pin1 expression in cervical cancer tissues as well as cell lines (HeLa, Siha, C33a, Caski) were examined (27). Pin1 was strikingly overexpressed in cervical cancers compared with the normal cervical tissues. Consistently, the expression levels of Pin 1 in all four human cancer cell lines examined were also higher than that in normal cervical epithelium. These results indicated that Pin1 overexpression was prevalent and specific in cervical cancers and contributed to oncogenesis of uterine cervix. Furthermore, we also found that the expression of Pin 1 increased progressively along with the disease process from normal cervix to CIN and to invasive cervical cancer $(\mathrm{p}<0.05)$. However, Pin 1 protein levels in invasive cervical cancers were not positively correlated with any commonly used clinicopathologic parameters, such as FIGO stage, pathological grade and 


\section{BamH It Target sense sequence Hairpin loop Target antisense sequence Terminator Top strand 5 -GATCCG CGGCTACATCCAGAAGATC TIAAGAGA GATCTTCTGGATGTAGCCG TTTTTT GGAAG-3' Bottom strand $\quad 3$ '-GC GCCGATGTAGGTCTTCTAG AAGTTCTCT CTAGAAGACCTACATCGGC AAAAAA CCTTCTTAA-5' EcoR1}

Figure 1. The shRNA oligonucleotide sequence design. The arrow shows the site of restrictive digestion.

lymph node metastasis. These results were consistent with that of previous researches in oral squamous cell carcinoma and colorectal cancer $(28,29)$. Further study showed that Pin 1 levels signi-ficantly correlated with cyclin D1 expression in cervical cancer, which suggested that Pin1 overexpression might promote transformation and uncontrolled proliferation of cervical epithelial cells through enhancing cyclin D1 expression and speeding up the cell cycle. Therefore, we hypothesize that Pin 1 is involved in cervical oncogenesis by regulating cyclin D1.

In the present study, the Pin 1 expression was manipulated by application of a Pin1-directed shRNA and a sense Pin1 plasmid and then the levels of cyclin D1 expression were tested to evaluate the effects of Pin1 on cyclin D1 in cervical cancer cell lines. Whether silencing Pin1 by RNAi blocked cell proliferation and induces apoptosis were investigated. Here we show that Pin1 overexpression might increase the expression of endogenous cyclin D1 in cervical cancer. Silencing Pin1, achieved by RNAi, was able to block cell proliferation and induce apoptosis in HeLa cells.

\section{Materials and methods}

Human cervical cancer cell line. HeLa cells were purchased from American Type Culture Collection (ATCC). Cells were cultured in DMEM with $10 \%$ fetal bovine serum.

Antibodies. Polyclonal rabbit anti-Pin1 (H-123), monoclonal mouse anti-cylin D1 antibodies were purchased from Santa Cruz, USA. RNAi-Ready pSIREN-DNR-DsRed-Express Vector and pEGFP-C1 Vector were purchased from BD Biosciences Clontech, USA.

shRNA plasmids construction. The sequence of Pin 1 mRNA was obtained from the GeneBank (No. BC002899). The Pin1 shRNA was designed by the web tools on Invitrogen homepage. The 297-315 bases of the Pin1 mRNA were chosen as the blocking target. The designed oligonucleotide to generate shRNA was synthesized by Invitrogen, USA (Fig. 1). The corresponding oligonucleotides were resuspended in TE buffer to a concentration of $100 \mu \mathrm{M}$. Briefly, the oligos for the top strand and the bottom strand were mixed at a 1:1 ratio then heated to $95^{\circ} \mathrm{C}$ for $30 \mathrm{sec}$ to remove all secondary structure, and then heated at $72^{\circ} \mathrm{C}$ for $2 \mathrm{~min}, 37^{\circ} \mathrm{C}$ for $2 \mathrm{~min}$, $25^{\circ} \mathrm{C}$ for $2 \mathrm{~min}$, and stored on ice. Then the annealed oligos were diluted with TE buffer to obtain a concentration of $0.5 \mu \mathrm{M}$. Following a ligation reaction the reaction mixture was incubated for $3 \mathrm{~h}$ at room temperature. Finally, the double strand oligos generating Pin1 shRNA were cloned into the RNAi-Ready pSIREN-DNR-DsRed-Express Vector.
Similarly, a negative control vector was constructed, and the recombinant plasmids were named pSIREN-PIN1 and pSIREN-Con, respectively.

Sense Pin1 plasmid construction. The CDS of Pin1 mRNA was amplified from HeLa cells with RT-PCR (Fig. 2A). The PCR primers for positive Pin1 were designed by Oligo software. The sequence of the upper primer was 5'-AGGGAATT CGATGGCGGACGAGGAGAAGCTGC-3' and the lower primer sequence was 5'-CATGGATCCGCTCCCCACCCTC ACTCAGTGC-3'. PCR conditions were as follows: 5 min for pre-denaturation at $94^{\circ} \mathrm{C}$, then $94^{\circ} \mathrm{C}$ for $30 \mathrm{sec}, 58^{\circ} \mathrm{C}$ for $60 \mathrm{sec}$ and $72^{\circ} \mathrm{C}$ for $90 \mathrm{sec}$ for 30 cycles. The PCR product (522 bp) was purified and restrictively digested with BamH1 and EcoR1, then cloned into pEGFP-C1 to form pEGFP-C1-Pin1 ${ }^{+}$. The target gene was in frame with the EGFP coding sequences, with no intervening in-frame stop codons.

Cell culture and transfection. HeLa cells were cultured in DMEM, supplemented with $10 \%$ FCS. Twenty-four hours before transfection, $3 \times 10^{5}$ cells were seeded onto 6-well plates and incubated at $37^{\circ} \mathrm{C}$ in $5 \% \mathrm{CO}_{2}$. HeLa cells grown to $80-95 \%$ confluence were transfected with $4 \mu \mathrm{g}$ of plasmid DNA and $10 \mu \mathrm{l}$ of Lipofectamine ${ }^{\mathrm{TM}} 2000$ (Invitrogen, USA) and DMEM media according to the manufacturer's recommendations. HeLa cells transfected with pSIREN-Con, pSIREN-PIN1, pEGFP-C1 and pEGFP-C1-Pin1 ${ }^{+}$, were named $\mathrm{HeLa} / \mathrm{p}-\mathrm{Con}, \mathrm{HeLa} / \mathrm{p}$-shRNA, HeLa/G-Con and HeLa/Pin ${ }^{+}$, respectively. Forty-eight hours after transfection, the brightness of fluorescence in HeLa cells was detected using fluorescence microscopy directly.

Western blot analysis. HeLa, HeLa/p-Con, HeLa/p-shRNA, $\mathrm{HeLa} / \mathrm{G}-\mathrm{Con}$ and $\mathrm{HeLa} / \mathrm{Pin} 1^{+}$cells extracts were prepared by detaching the adherent cells with trypsin/EDTA, triplicate washing with PBS. To detect Pin1 levels using Western blotting analysis, cell lysates were obtained by sonication of cell pellets in $50 \mathrm{mmol} / \mathrm{l}$ Tris- $\mathrm{HCl}, \mathrm{pH} 8.0,150 \mathrm{mmol} / 1 \mathrm{NaCl}$, $0.5 \%$ Triton $\mathrm{X}-100,10 \mu \mathrm{g} / \mathrm{ml}$ phenylmethyl sulfonyl fluoride, and $20 \mu \mathrm{g} / \mathrm{ml}$ leupeptin as described $(9,10,11,27)$. Lysates were clarified by centrifugation $12,000 \mathrm{~g}$ at $4^{\circ} \mathrm{C}$ for $30 \mathrm{~min}$. Proteins were resolved by $12 \%$ sodium dodecyl sulfatepolyacrylamide gel electrophoresis, then were transferred at $4^{\circ} \mathrm{C}$ in $1 \mathrm{x}$ Tris-glycine-sodium dodecyl sulfate containing $20 \%$ methanol onto nitrocellulose. Immunoblotting was performed with anti-Pin1, anti-cyclin D1 or anti-actin antibodies as described $(9,10)$. Bound antibodies were detected by ECL (Biological Industries kibbutz, Beit Haemek, Israel). Levels of Pin 1, cyclin D1 and actin were quantified by densitometry using Imagequant software, followed by expressing Pin1 levels 


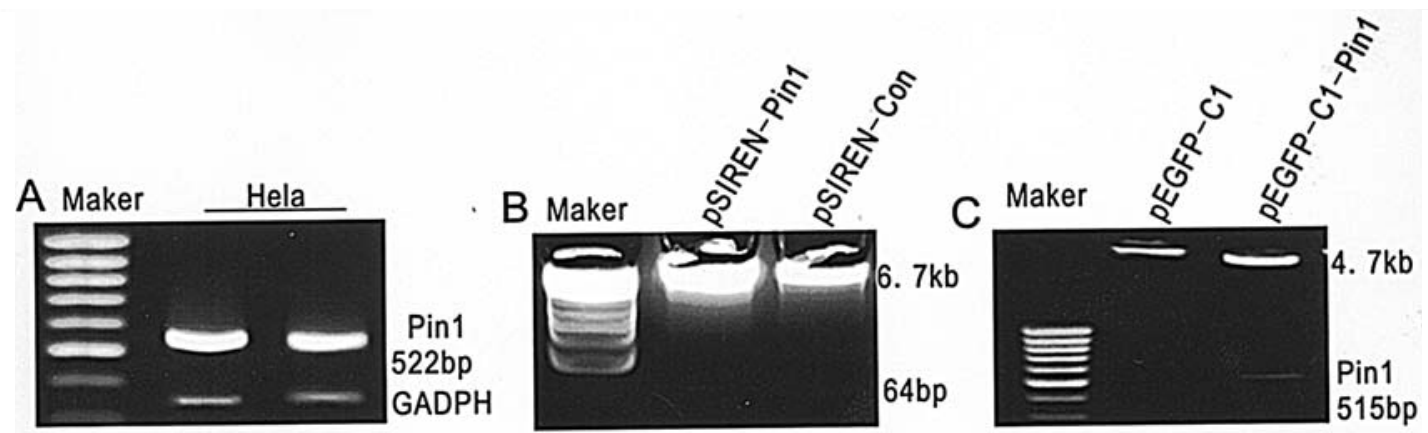

Figure 2. Construction and identifying of the recombinant plasmids. (A), The CDS of Pin1 mRNA was amplified from HeLa cells by RT-PCR (522 bp). (B), The plasmid pSIREN-PIN1 or pSIREN-Con with the restrictive digestion showed two bands after electrophoresis for 5 min. (C), pEGFP-C1 showed a single band $(4.7 \mathrm{~kb})$ in the gel. pEGFP-C1-Pin $1^{+}$showed two bands in the gel (intervened DNA was $515 \mathrm{bp}$, the vector was $4.7 \mathrm{~kb}$ ).

as Pin1:actin ratios, cyclin D1 as cyclin D1:actin, as described $(9,10)$. The average ratios were obtained from multiple gels.

Colony formation experiments in soft agar. HeLa cells were plated in $0.3 \%$ soft agar at $24 \mathrm{~h}$ after transfection with pSIRENCon or pSIREN-PIN1. The colonies were counted in three different wells in two independent experiments at 15 days after transfection, and the averages were plotted.

MTT assay. HeLa cells $\left(5 \times 10^{3}\right)$ were plated in 96 -well plates in DMEM containing 10\% NBS in a final volume of $0.2 \mathrm{ml}$ after being transfected for $12 \mathrm{~h}$. When cells were cultured for 12, 24, 48, 72 and $96 \mathrm{~h}$, MTT was added with $20 \mu \mathrm{l}$ per well. When incubated at $37^{\circ} \mathrm{C}$ for $4 \mathrm{~h}$, the reaction was blocked by the addition of $150 \mu 1$ of DMSO. After being shaken for 15 min, cell proliferation was assessed by detection of the absorbence of the samples on $\mu$ Quant (Bio-Tekinstruments, Inc.) at $570 \mathrm{~nm}(\mathrm{~A} 570)$.

Flow cytometry. HeLa cell pellets were prepared by detaching the adherent cells with trypsin/EDTA $48 \mathrm{~h}$ after transfected, washing with PBS, and were fixed in ice-cold ethanol (75\%) at $-20^{\circ} \mathrm{C}$ for several days. Then the fixed cells were pelleted, washed three times with PBS, digested with $10 \mu \mathrm{g} / \mathrm{ml}$ RNase for $30 \mathrm{~min}$ at $37^{\circ} \mathrm{C}$, and stained with $50 \mu \mathrm{g} / \mathrm{ml}$ PI solutions for $1 \mathrm{~h}$ at $4^{\circ} \mathrm{C}$. The DNA content was analyzed and the percentage of apoptotic cells was calculated using a FACS (BD, USA). Experiments were performed in triplicate.

Statistical analysis. All statistical analysis was performed using SPSS 11.5 software. One-way ANOVA analysis was used to evaluate the differences among groups. The results of the twosided test were considered significant when $\mathrm{p}<0.05$.

\section{Results}

Identification of the recombinant plasmids. The E. coli was transformed with recombinant plasmid DNA. As plasmids pSIREN-PIN1 and pSIREN-Con carried an ampicillin resistance gene, the E. coli transformed with pSIREN-PIN1 or pSIREN-Con grew on a selective media containing ampicillin. Similarly, plasmids pEGFP-C1 and pEGFP-C1Pin $1^{+}$carried a kanamycin resistance gene, the $E$. coli transformed with pEGFP-C1 or pEGFP-C1-Pin1+ grew on a selective media containing kanamycin. The transformed E.coli were cultured and amplified in the media containing ampicillin or kanamycin, respectively. Plasmid DNAs were digested with restriction enzymes and then analyzed by electrophoresis. The restriction digest of pSIREN-PIN1 or pSIREN-Con separated into two bands in the gel (intervened DNA was $64 \mathrm{bp}$, and vector was $6.7 \mathrm{~kb}$ ), (Fig. 2B). The restriction digest of pEGFP-C1 was a single band $(4.7 \mathrm{~kb})$, and the pEGFP-C1-Pin $1^{+}$had two bands (intervened DNA was $515 \mathrm{bp}$, and vector was $4.7 \mathrm{~kb}$ ), (Fig. 2C). DNA sequencings was done at the same time. Sequencing showed that the vectors of pSIREN-PIN1 and pSIREN-Con were completively corrected, while the pEGFP-C1-Pin $1^{+}$had lost 6 bp from 82nd to 87th, encoding serine (19th) and glycine (20th). By blasting on Pubmed and predicting its high structure, the above two amino acids were coincidently the variable site and their loss had no effect on the function of Pin1. The two amino acids were located in the WW domain of Pin1. The Pin1-WW domain was just required for the localization of Pin1 into nuclear speckles. Once the Pin1-WW domain was abnormal, GFP distributed throughout the cell and showed no localization in nuclear speckles (30). In our present experiments, we clearly identified the nuclear speckles with fluorescent microscopic inspection after transfection with pEGFP- C1-Pin $1^{+}$in HeLa cells (Fig. 3D), as also shown previously (30).

Transfection and effects of the recombinant plasmids. HeLa cells transfected with pSIREN-PIN1, which generate Pin1 shRNA expresses a variant of Discosoma sp. red fluorescence (Fig. 3). HeLa cells transfected with pEGFP-C1-Pin 1+ expresses a red-shifted variant of wild-type GFP. And it has been optimized so that the brighter the fluorescence, the higher the expression levels of the respective protein in HeLa cells.

Then the PIN1 mRNA and protein expression were detected by RT-PCR and Western blot. Pin1 shRNA significantly reduced the expression levels of PIN1 mRNA and protein. After transfected with pSIREN-PIN1 for $48 \mathrm{~h}$, the ratio of PIN1 mRNA/GAPDH mRNA was $0.19 \pm 0.05$. Compared with $0.84 \pm 0.16$ in $\mathrm{HeLa} / \mathrm{p}-\mathrm{Con}$, the expression of PIN1 mRNA was inhibited by $77.38 \%(\mathrm{p}<0.05)$. The ratio of Pin 1 protein/ß-actin protein was $0.33 \pm 0.14$ and $0.79 \pm 0.17$ in $\mathrm{HeLa} / \mathrm{p}$-shRNA and $\mathrm{HeLa} / \mathrm{p}-\mathrm{Con}$, respectively. At the protein level, Pin1 was 

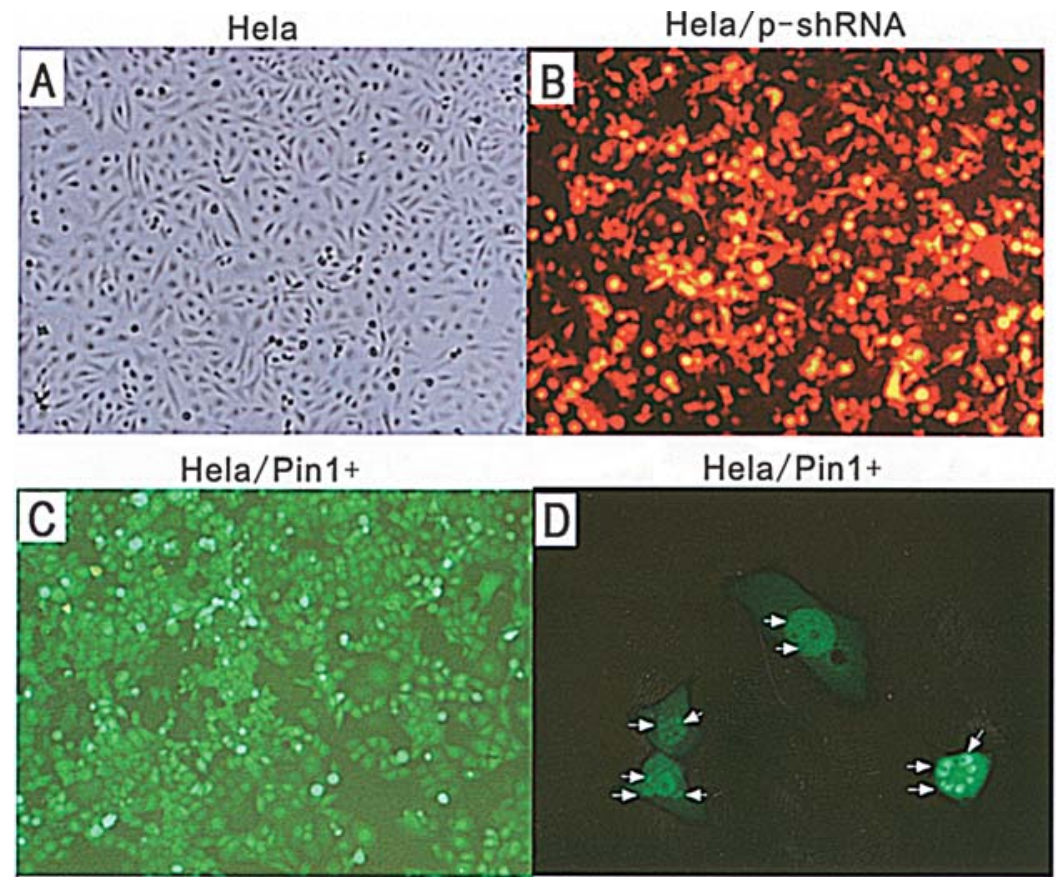

Figure 3. Fluorescent imaging of the HeLa cells transfected with recombinant vectors. (A), HeLa cells (x100). (B), HeLa/p-shRNA, HeLa cells transfected with pSIREN-PIN1 which generate Pin1 shRNA (x100). The recombinant pSIREN-PIN1 constitutively expresses a variant of Discosoma sp. red fluorescent protein. (C and D), HeLa/Pin 1 ${ }^{+}$, HeLa cells transfected with pEGFP-C1-Pin $1^{+}$which generate Pin1. The pEGFP-C1-Pin $1^{+}$encodes a red-shifted variant of wildtype GFP, which has been optimized so that the brightness of fluorescence indicates the expression levels in HeLa cells (C, x100). GFP-tagged Pin1 subcellular localization was analyzed by fluorescent microscopic inspection (D, x800). As shown in (D), Pin1 localizes almost exclusively in the cell nucleus speckles and concentrate at discrete structures.

inhibited by $58.23 \%(\mathrm{p}<0.05)$. The plasmid of pEGFP-C1-Pin $1^{+}$ could generated GFP-tagged Pin1. Comparing with the HeLa/ G-Con, PIN1 mRNA and protein were significantly increased $48 \mathrm{~h}$ after transfection in HeLa/Pin $1^{+}$.

Cyclin D1 expression levels in HeLa cells after manipulation of Pin1. Given the positive correlation between Pin1 and cyclin D1 protein expression in cervical cancer tissues (27), we were interested in determining whether Pin 1 overexpression might increase the expression of endogenous cyclin D1 in cervical cancer. To examine this possibility, we transiently transfected a Pin1 expression construct (pEGFP-C1-Pin $1^{+}$) into HeLa cells, and examined the changes of endogenous cyclin D1 expression levels. Pin1 overexpression led to the elevation of the cyclin D1 protein expression levels in HeLa cells (Fig. 4). Moreover, to elucidate the effect of Pin1 depletion on cyclin D1 expression, HeLa cells were transiently transfected with the control vector or a construct expression shRNA which can interfere with PIN1 mRNA (pSIRENPIN1), followed by immunoblotting analysis with anti-Pin1, cyclin D1 or B-actin antibodies $48 \mathrm{~h}$ after transfection. Silencing Pin1 significantly reduced the levels of cyclin D1 protein expression in HeLa cells (Fig. 4). Thus, Pin1 levels could affect the expression of cyclin D1 in HeLa cells.

Silencing Pinl blocks HeLa cell proliferation. Functionally, Pin1 is critical for cell proliferation in vivo $(1,2,5,11,30)$. Phosphorylation-dependent proline isomerization catalyzed by Pin 1 is essential for tumor cell survival and entry mitosis in vitro $(30,31)$. In the present study, Pin 1 was specifically suppressed by RNA interference, and then its effects on the proliferation of HeLa cells were explored. The effect of
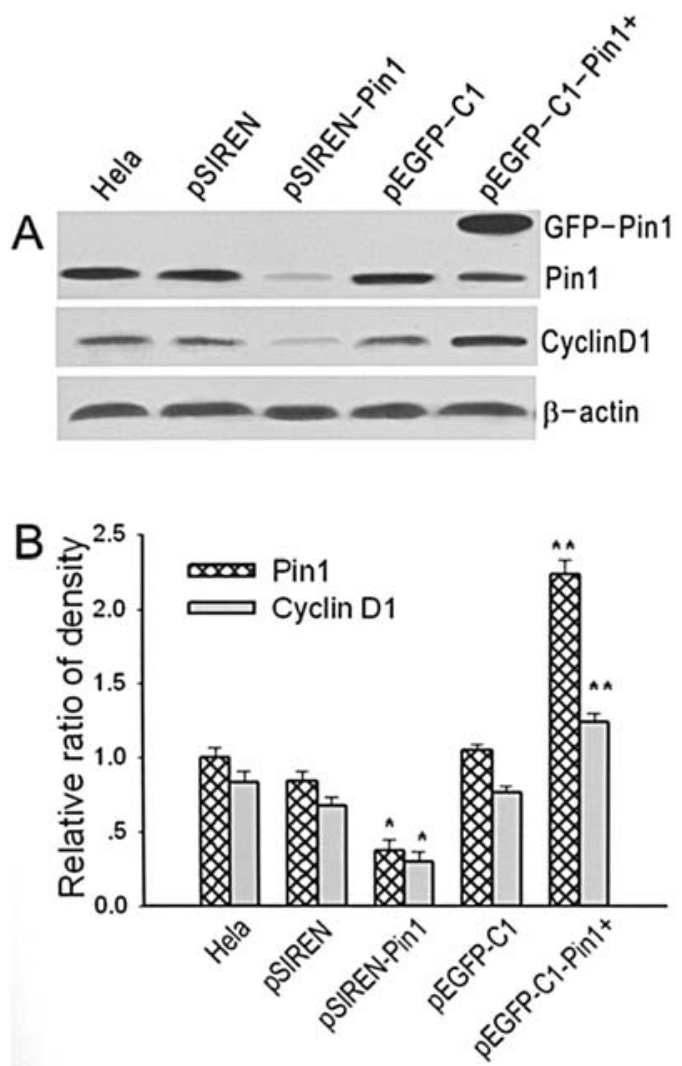

Figure 4. Manipulation of Pin 1 caused changes of cyclin D1 expression levels in HeLa cells. (A), HeLa, cells analyzed with immunoblotting $48 \mathrm{~h}$ after transient transfection with pSIREN, pSIREN-PIN1, pEGFP-C1 and pEGFPC1-Pin $1^{+}$respectively. (B), Overexpression of Pin1 (pEGFP-C1-Pin $1^{+}$) significantly increased the expression levels of cyclin D1 protein in HeLa cells $\left({ }^{* *} \mathrm{p}<0.05\right)$. In contrast, depletion of Pin 1 (pSIREN-PIN1) significantly reduced the expression levels of cyclin D1 protein in HeLa cells $\left({ }^{*} \mathrm{p}<0.05\right)$. 

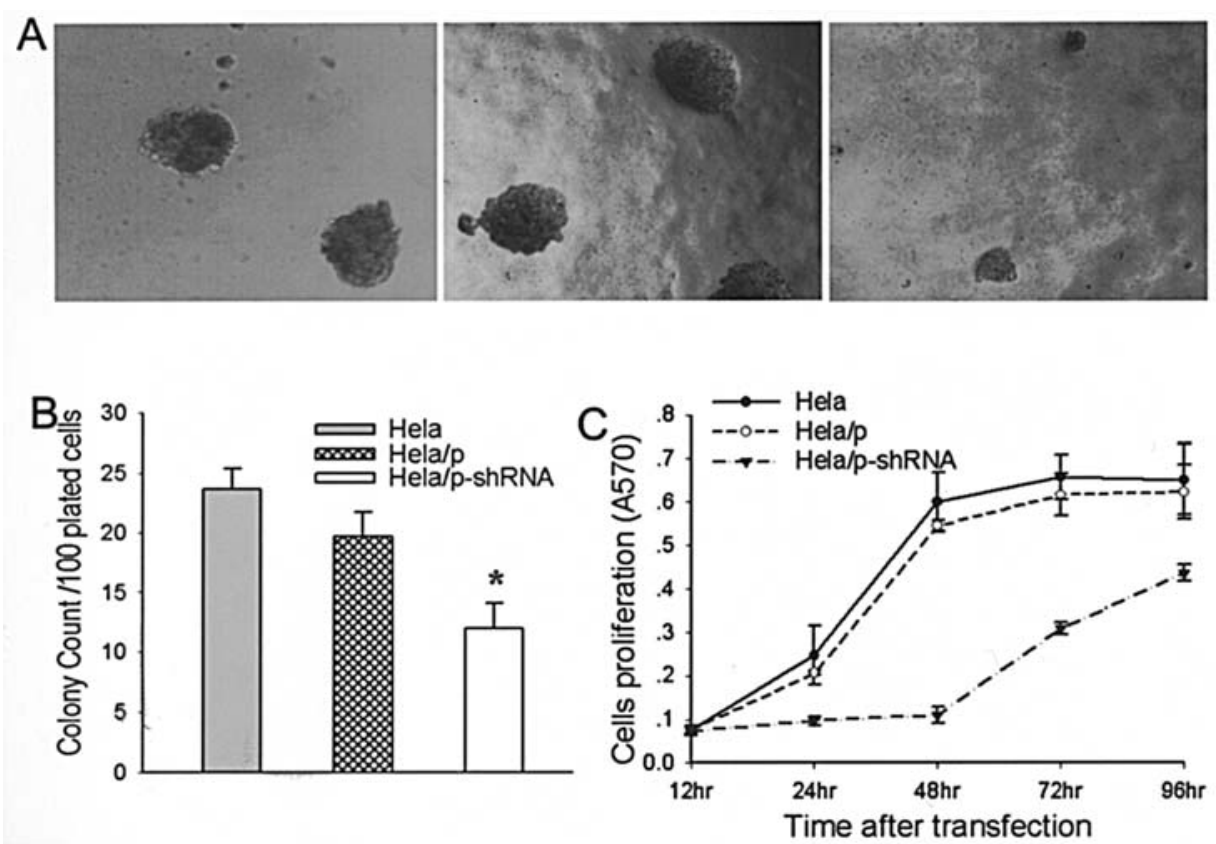

Figure 5. Effects of Pin1 shRNA on colony formation (A and B) and proliferation (C) of HeLa cells. (A and B), HeLa and HeLa/p-Con cells [(A), the left and the middle] could form many colonies in soft agar, and the colonies above were rounder and larger than that in HeLa/p-shRNA cells [(A), on the right]. The reduction in the number of colonies in the HeLa/p-shRNA group was statistically significant compared with that in the HeLa or HeLa/p-Con groups, $\left({ }^{*} \mathrm{p}<0.05\right.$, t-test). (C), Proliferation of HeLa cells was detected with MTT assay. HeLa cells and HeLa cells transfected with pSIREN-Con or pSIREN-PIN1 were reseeded in 96-well plates. The value of A570 was considered as the levels of cell proliferation. HeLa/p-shRNA cells growth was suppressed significantly $(\mathrm{p}<0.05)$.
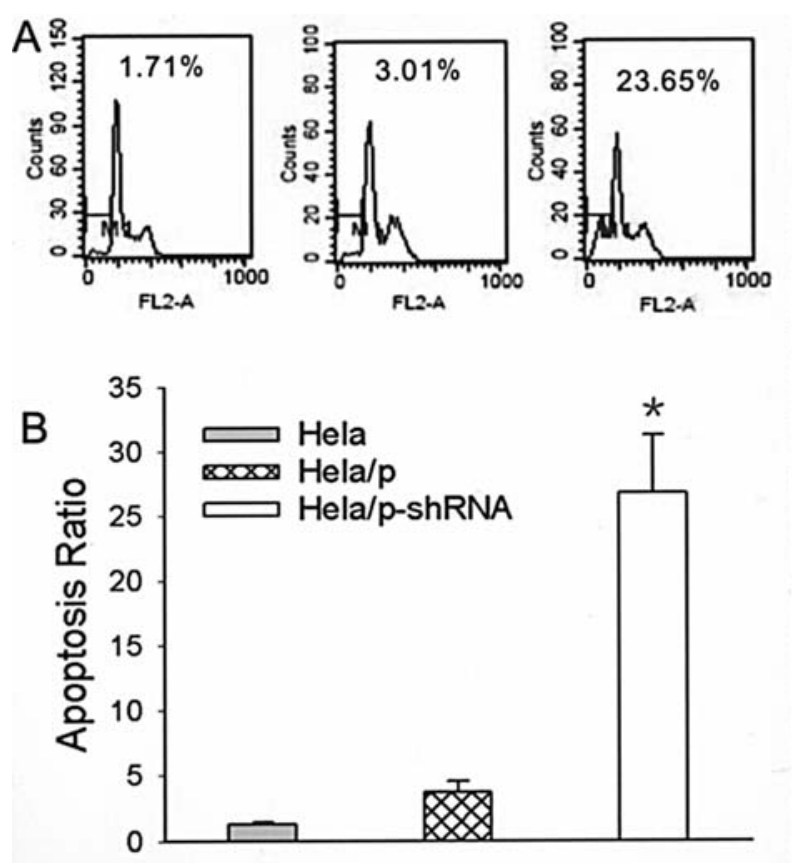

Figure 6. Silencing Pin1 induces the apoptosis of HeLa cells. (A), Apoptosis of the three cells groups were analyzed with flow cytometry $48 \mathrm{~h}$ after transfection. (A, left) HeLa cells. (A, middle) HeLa/p-Con cells (HeLa transiently transfected with p-SIREN-Con). (A, right) HeLa/p-shRNA cells (HeLa transiently transfected with p-SIREN-PIN1). (B), The ratio of apoptosis in HeLa/p-shRNA was significantly higher than that in HeLa and $\mathrm{HeLa} / \mathrm{p}-\mathrm{Con}$ cells. Average apoptotic ratio of three independent experiments is shown: bars, $\mathrm{SE} ;{ }^{*} \mathrm{p}<0.05, \chi^{2}$ test.

suppressing Pin 1 on cell growth was determined by the colony formation test. As shown in Fig. 5A and B, HeLa and $\mathrm{HeLa} / \mathrm{p}$-Con cells formed many colonies in soft agar, and the colonies above were rounder and larger than the HeLa/pshRNA colonies. The colony ratios (mean colony count/ 100 plated cells \pm SD) were as follows: HeLa, 23.67\% \pm 4.27 ; $\mathrm{HeLa} / \mathrm{p}-\mathrm{Con}, 19.67 \% \pm 4.97 ; \mathrm{HeLa} / \mathrm{p}-\mathrm{shRNA}, 12.00 \% \pm 5.21$ $(\mathrm{p}<0.05)$. The results of MTT assay exhibited a similar proliferation status (Fig. 5C).

Silencing Pin1 induces apoptosis in HeLa cells. Pin1 deletion induced apoptosis and prevented cells from entering mitotic phase $(29,30)$. RNA interference by Pin 1 shRNA was used in this study, and then the effects on cell apoptosis was examined by FAC Scan analysis. The ratio of apoptosis was $26.76 \% \pm 7.42$ in $\mathrm{HeLa} / \mathrm{p}$-shRNA cell, which was significantly higher than that in HeLa $(1.33 \pm 0.15)$ and HeLa/p-Con cell $(3.73 \pm 0.78)$ $(\mathrm{p}<0.05)$ (Fig. 6).

\section{Discussion}

In the present study, to further verify the possible mechanism of Pin1 in cervical cancer, some recombinant plasmids were first constructed and the Pin1 expression levels were tested. Then the effects of Pin1 on cyclin D1 expression were explored in cervical cancer cell lines. HeLa cells were transiently transfected with a sense plasmid of Pin1. With the increasing of the cellular Pin 1 expression, cyclin D1 level increased in HeLa cells. In contrast, Pin1 deletion specifically with RNAi technique obviously suppressed the expression of cyclin D1. These results suggested that Pin1 could directly regulate the activity of cyclin D1 in human cervical cancer. This is significant given that cyclin D1 is an essential downstream target for cervical tumorigenesis. It has been shown that, as a catalyst, Pin 1 contributes to cervical tumorigenesis. One of mechanisms of Pin1 is to regulate cyclin D1 expression in human cervical cancer. Practically, Pin1 can enhance cyclin 
D1 expression by activated transcription and post-transcriptional stability in breast cancer. Up to now, it has not been reported why Pin1 is overexpressed in cervical cancer. It is worthwhile to investigate this in the future.

As the catalytic activity of Pin 1 is essential for tumor cell survival, it is indicated that the inhibition of Pin1 expression can suppress oncogenesis, offering a new option for anticancer therapy (30-32). Previous results have shown that Pin 1 deletion could induce apoptosis of cancer cells; overexpression of Pin1 antisense RNA or dominant-negative Pin1, and application of a known small-molecule Pin1-PPIase inhibitor, juglone prevented the cancer cells from entering mitosis $(30,33)$. However, the feasibility of therapeutic Pin 1 inhibition has not yet been explored because of the lack of Pin1-specific inhibitors. Therefore, there is a need for the development of Pin1-specific deletion. In the present study, a new technique, RNA interference (RNAi) was used to specifically suppress Pin1. The results showed that silencing Pin 1, achieved by RNAi, obviously blocked the cell proliferation and induced apoptosis of HeLa cells. The above results demonstrated that Pin1 plays an important role during oncogenesis and promote tumor growth in cervical cancer. Furthermore, it is also suggested that Pin1 may serve as a diagnostic marker and also as a novel therapeutic target for human cervical cancer $(32,34)$.

In conclusion, we showed that Pin 1 is overexpressed in cervical cancer and contributes to cervical tumorigenesis by increasing the cyclin D1 expression level. Silencing Pin1 with RNAi blocked cell proliferation and induced apoptosis of cervical cancer cells along with decreasing the cyclin D1 expression level. Taken together, our study suggested that Pin1 may be a critical catalyst for oncogenesis by regulating the cyclin D1 activity, and overexpressed Pin1 may act as a novel molecular marker for diagnostics and be a specific target for therapeutics in human cervical cancer.

\section{Acknowledgements}

Grants support: National Science Foundation of China (No. 30528012; 30271358) and the '973' Program of China (No. 2002CB513100).

\section{References}

1. Lu KP, Hanes SD and Hunter T: A human peptidyl-prolyl isomerase essential for regulation of mitosis. Nature 380: 544-547, 1996.

2. Yaffe MB, Schutkowski M, Shen M, et al: Sequence-specific and phosphorylation-dependent proline isomerization: a potential mitotic regulatory mechanism. Science 278: 1957-1960, 1997.

3. Ranganathan R, Lu KP, Hunter T and Noel JP: Structural and functional analysis of the mitotic peptidyl-prolyl isomerase Pin1 suggests that substrate recognition is phosphorylation dependent. Cell 89: 875-886, 1997.

4. Lu KP, Liou YC and Zhou XZ: Pinning down the prolinedirected phosphorylation signaling. Trends Cell Biol: 164-172, 2002.

5. Lu KP: Phosphorylation-dependent prolyl isomerization: a novel cell cycle regulatory mechanism. Prog Cell Cycle Res 4: 83-96, 2000.

6. Blume-Jensen P and Hunter T: Oncogenic kinase signalling. Nature 411: 355-365, 2001.

7. Lu KP: Pinning down cell signaling, cancer and Alzheimer's disease. Trends Biochem Sci 29: 200-209, 2004.

8. Yeh E, Cunningham M, Arnold $\mathrm{H}$, et al: A signalling pathway controlling c-Myc degradation that impacts oncogenic transformation of human cells. Nat Cell Biol 6: 308-318, 2004.
9. Bao L, Kimzey A, Sauter G, Sowadski JM, Lu KP and Wang DG: Prevalent overexpression of prolyl isomerase Pin1 in human cancers. Am J Pathol 164: 1727-1737, 2004.

10. Wulf GM, Ryo A, Wulf GG, et al: Pin1 is overexpressed in breast cancer and cooperates with Ras signaling in increasing the transcriptional activity of c-Jun towards cyclin D1. EMBO J 20: 3459-3472, 2001.

11. Ayala G, Wang D, Wulf G, et al: Pin1 is a novel prognostic marker in prostate cancer. Cancer Res 63: 6244-6251, 2003.

12. Wulf G, Garg P, Liou YC, Iglehart D and Lu KP: Modeling breast cancer in vivo and ex vivo reveals an essential role of Pin1 in tumorigenesis. EMBO J 23: 3397-3407, 2004.

13. Hongyu LI and Ding MA: A Catalyst for oncogenesis: peptidylprolyl cis/trans isomerase Pin1. J Med Mol Biol 2: 292-294, 2005.

14. Yu Q, Geng Y and Sicinski P: Specific protection against breast cancers by cyclin D1 ablation. Nature 411: 1017-1021, 2001.

15. Lamb J, Ramaswamy S, Ford HL, et al: A mechanism of cyclin D1 action encoded in the patterns of gene expression in human cancer. Cell 114: 323-334, 2003.

16. Ryo A, Nakamura M, Wulf G, Liou YC and Lu KP: Pin1 regulates turnover and subcellular localization of $\beta$-catenin by inhibiting its interaction with APC. Nat Cell Biol 3: 793-801, 2001.

17. Liou YC, Ryo A, Huang HK, et al: Loss of Pin1 function in the mouse causes phenotypes resembling cyclin D1-null phenotypes. Proc Natl Acad Sci USA 99: 1335-1340, 2002

18. Ryo A, Suizu F, Yoshida Y, et al: Regulation of NF-kappaB signaling by Pin1-dependent prolyl isomerization and ubiquitinmediated proteolysis of p65/RelA. Mol Cell 12: 1413-1426, 2003.

19. Gao M, Labuda T, Xia Y, Gallagher E, Fang D, Liu YC and Karin M: Jun turnover is controlled through JNK dependent phosphorylation of the E3 ligase Itch. Science 306: 271-275, 2004.

20. Moon RT, Bowerman B, Boutros M and Perrimon N: The promise and perils of Wnt signaling through $\beta$-catenin. Science 296: 1644-1646, 2002.

21. Bienz M: The subcellular destinations of APC proteins. Nat Rev Mol Cell Biol 3: 328-338, 2002.

22. Pang R, Yuen J, Yuen MF, et al: PIN1 overexpression and Bcatenin gene mutations are distinct oncogenic events in human hepatocellular carcinoma. Oncogene 23: 4182-4186, 2004.

23. Karin M, Cao Y, Greten FR and Li ZW: NF-кB in cancer: from innocent bystander to major culprit. Nat Rev Cancer 2: 301-310, 2002.

24. Wulf G, Finn G, Suizu F and Lu KP: Phosphorylation-specific prolyl isomerization: is there an underlying theme? Nat Cell Biol 7: 435-441, 2005.

25. Bishop JM: Molecular themes in oncogenesis. Cell 64: 235-248, 1991.

26. Ryo A, Liou YC, Wulf G, Nakamura M, Lee SW and Lu KP: Pin 1 is an E2F target gene essential for the Neu/Ras-induced transformation of mammary epithelial cells. Mol Cell Biol 22: 5281-5295, 2002

27. Li HY, Xu Q, Zhu Tao, Zhou JH, Deng DR, Wang SX, Lu YP and Ma D: Expression of Pin1, cyclin D1 in cervical cancer and its significance. Ai Zheng 25: 367-372, 2006.

28. Miyashita H, Mori S, Motegi K, Fukumoto M and Uchida T: Pin1 is overexpressed in oral squamous cell carcinoma and its levels correlate with cyclin D1 overexpression. Oncol Rep 10: 455-461, 2003.

29. Kim CJ, Cho YG, Park YG, et al: Pin1 overexpression in colorectal cancer and its correlation with aberrant $\beta$-catenin expression. World J Gastroenterol 11: 5006-5009, 2005

30. Rippmann JF, Hobbie S, Daiber C, et al: Phosphorylationdependent proline isomerization catalyzed by Pin 1 is essential for tumor cell survival and entry into mitosis. Cell Growth Differ 11: 409-416, 2000.

31. Winkler KE, Swenson KI, Kornbluth S and Means AR: Requirement of theprolyl isomerase Pin 1 for the replication check-point. Science 287: 1644-1647, 2000.

32. Ryo A, Liou YC, Lu KP and Wulf G: Prolyl isomerase Pin1: a catalyst for oncogenesis and a potential therapeutic target in cancer. J Cell Sci 116: 773-783, 2003.

33. Uchida T, Takamiya M, Takahashi M, et al: Pin1 and Par14 peptidyl prolyl isomerase inhibitors block cell proliferation. Chem Biol 10: 15-24, 2003.

34. Lu KP: Prolyl isomerase Pin1 as a molecular target for cancer diagnostics and therapeutics. Cancer Cell 4: 175-180, 2003. 